\title{
Transmit and Receive Filter Design For OFDM Based WLAN Systems
}

\author{
Pei Xiao, Ciaran Toal, Dwayne Burns, Vicent Fusco, Colin Cowan \\ The Institute of Electronics, Communications and Information Technology \\ Queen's University Belfast, BT3 9DT, United Kingdom \\ E-mail: pei.xiao@ecit.qub.ac.uk
}

\begin{abstract}
We analyze the effect of different pulse shaping filters on the orthogonal frequency division multiplexing (OFDM) based wireless local area network (LAN) systems in this paper. In particular, the performances of the square root raised cosine (RRC) pulses with different rolloff factors are evaluated and compared. This work provides some guidances on how to choose RRC pulses in practical WLAN systems, e.g., the selection of rolloff factor, truncation length, oversampling rate, quantization levels, etc..
\end{abstract}

\section{INTRODUCTION}

Wireless local area network (WLAN) has experienced tremendous growth in the last decade. It provides cable-free, high data rates access in both indoor and outdoor environments. A WLAN offers a very cost effective way of building an access network due to its easy maintainability, low incremental cost and portability. The physical layer of 802.11a WLAN system [1] is based on orthogonal frequency division multiplexing (OFDM) [2], a modulation technique that uses multiple carriers to mitigate the effects of multipath. OFDM distributes the data over a large number of carriers that are spaced apart at precise frequencies.

Most work on OFDM systems only considers the use of rectangular window to simplify the system design and reduces the computational complexity. However, more spectrally efficient pulses are employed in practical systems in order to satisfy the bandwidth limitations imposed by the channels and to limit the out-of-band power. Square root raised cosine (RRC) pulses have been proposed for transmit and receive filters in various industrial standards, such as 802.11a WLAN system [1], wideband code division multiplex access (WCDMA) system [3], and 3GPP Long Term Evolution (LTE), also known as EvolvedUMTS Terrestrial Radio Access [4]. In this paper, we investigate different aspects of the RRC pulse filters in attempt to shed some light on the transmit and receive filter design for practical WLAN systems.

The transmitter and receiver diagrams of the considered WLAN system are shown in Fig. 1. At the transmitter, the information bit sequence is encoded into a

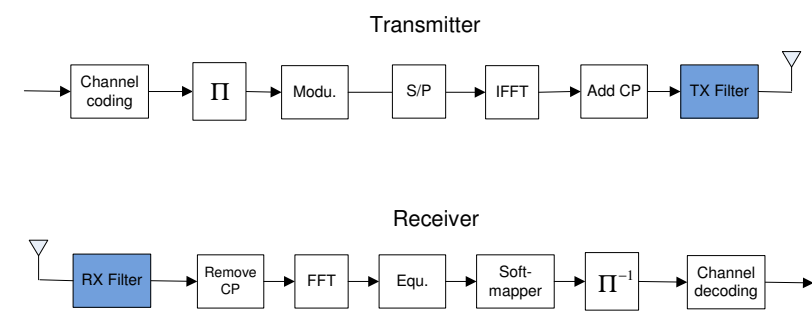

Fig. 1. Transmitter and receiver structures of the OFDM based WLAN system.

coded bit sequence which is interleaved and mapped into QPSK/QAM symbols. A set of $N$ symbols is serial to parallel converted and are imposed onto orthogonal sub-carriers by the means of inverse fast Fourier transform (IFFT). The output from IFFT block is converted into serial data and a cyclic prefix $(\mathrm{CP})$ is inserted to form one OFDM symbol for transmission. The length of the $\mathrm{CP}$ is assumed to be longer than the impulse response of the channel to combat intersymbol interference (ISI) and inter-carrier interference (ICI). The OFDM signal is filtered by a transmit pulse shaping filter and transmitted over the multipath fading channel. At the receiver, a receive pulse shaping filter is applied to the received signal. This process is followed by the $\mathrm{CP}$ removal and subsequent fast Fourier transform (FFT) operation to convert the signal back to the frequency domain. We then apply a one-tap equalizer to mitigate the channel effect. The output of the equalizer is fed to a soft demapper to derive soft estimates of the transmitted bits which are subsequently de-interleaved and decoded to recover the information bearing signal. In this work, we focus on the design of transmit and receive filters that are suitable for practical implementations.

\section{Comparison of Different Pulse Shaping FILTERS}

The impulse response of the investigated RRC filter is given by [5]

$$
g(t)=\frac{\sin [\pi t(1-\alpha) / T]+4 \alpha t \cos [\pi t(1+\alpha) / T] / T}{\pi t\left[1-(4 \alpha t / T)^{2}\right] / T}
$$



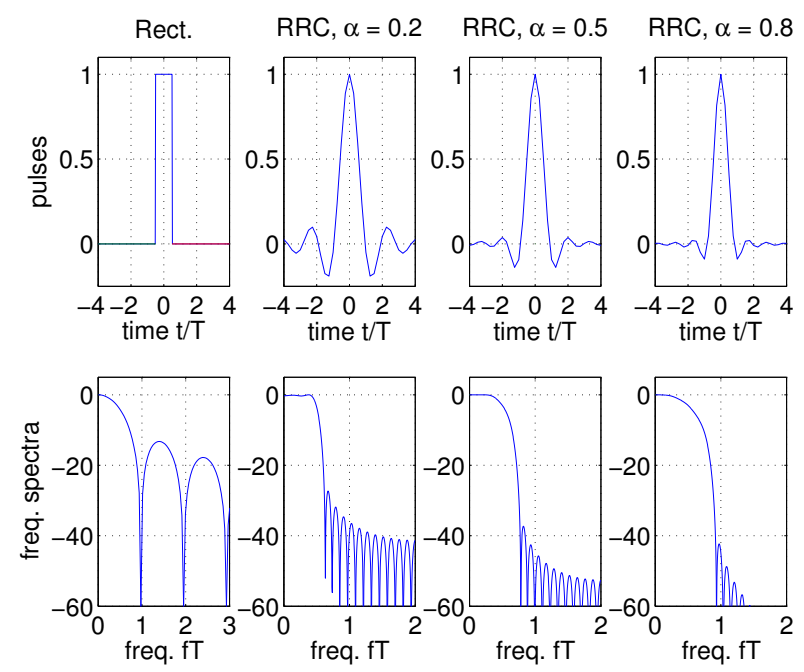

Fig. 2. Different pulses (upper plots) and their corresponding spectra (lower plots). The $\mathrm{x}$-axis for pulses is normalized to the symbol interval $T$, the frequency axis for spectra is normalized to the symbol rate $1 / T$, the magnitude of the spectra is plotted in $\mathrm{dB}$ scale. All pulses are normalized to a peak value of unity.

where $\alpha$ is the rolloff factor, $T$ is the symbol interval.

Different pulses employed in our simulations are illustrated in Fig. 2, where we use RRC pulses with rolloff factors $\alpha=0.2,0.5,0.8$, truncated to 4 symbol interval on each side of the peak $\left(T_{r}=4 T\right)$. Apparently, the rectangular pulse has the spectral deficiency of infinite bandwidth occupancy, which can be observed from its spectrum plot. Given the same truncation length, the sidelobe reduces in the case of RRC pulses, in the meantime, more excess bandwidth is introduced as rolloff factor increases.

The eye diagrams are useful visual tools for evaluating the performance of different pulse shapes. They are generated using an oscilloscope connected to the output of the receive filter and before the sampler. Therefore, what we see on the oscilloscope is the convolution of the pulse shaping filter $g(t) \otimes g(-t)$. The convolution of a RRC pulse becomes a raised cosine (RC) pulse. The oscilloscope is re-triggered at every symbol period. By relying on the persistence of a typical oscilloscope display, the result is an overlaying of consecutive received symbol waveforms which form an eye pattern on the screen. We re-create this effect with computer simulation and plot it in Fig. 3.

As demonstrated in the diagrams, the eye pattern of the RC pulse with $\alpha=0.8$ is more open compared to that of the RC pulses with $\alpha=0.2$ and $\alpha=0.5$. The RC pulse with $\alpha=0.8$ exhibits a larger margin against the ISI, ICI and additive noise, therefore it is more robust to synchronization errors than the other two RRC pulses.

In the investigated WLAN system, we use the
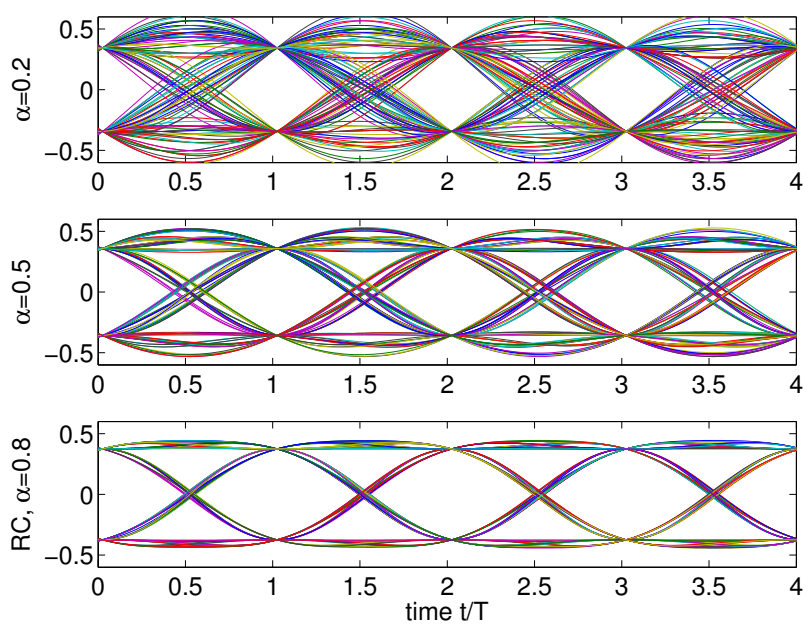

Fig. 3. Eye diagrams of employed pulse shapes.

convoluational code with constraint length of 7 and generator polynomial $(133,171)_{8}$. The FFT size is 1024 , and the length of CP is chosen to be 64. The modulation scheme is 16-QAM. We use the following channel model adopted by the IEEE 802.11 working group [6]

$$
\begin{aligned}
h_{k} & =N\left(0,0.5 \sigma_{k}^{2}\right)+j N\left(0,0.5 \sigma_{k}^{2}\right) ; \\
\sigma_{k}^{2} & =\sigma_{0}^{2} \exp \left(-k T_{s} / T_{R M S}\right) ; \\
\sigma_{0}^{2} & =1-\exp \left(-T_{s} / T_{R M S}\right),
\end{aligned}
$$

where $h_{k}$ is the complex channel gain of the $k$ th tap, $T_{R M S}$ is the RMS delay spread of the channel, $T_{s}$ is the sampling period, $\sigma_{0}^{2}$ is chosen so that the condition $\sum_{k} \sigma_{k}^{2}=1$ is satisfied to ensure same average received power. The number of samples to be taken in the impulse response should ensure sufficient decay of the impulse response tail, e.g., $k_{\max }=10 \times T_{R M S} / T_{s}$. The RMS delay spread is set to be $T_{R M S}=50 \mathrm{~ns}$ and the sampling rate $f_{s}=1 / T_{s}=100 \mathrm{MHz}$.

In Fig. 4, we measure the performance of different pulses versus oversampling rate $\eta$ to see what would be proper value of $\eta$ for the WLAN system under study. Apparently, $\eta$ has to be no less than 6 for all the pulses. The performance gets saturated when it goes beyond this point.

All the RRC pulses have infinite length. We have to do truncation to make them realizable. In practical systems, it would be desirable to have a shorter pulse in order to reduce the computational complexity. However, as illustrated in Fig. 5, shorter truncation length lead to larger sidelobe of the signal spectrum, or larger out-of-band power, which in turn will increase the ISI and degrade the system performance.

In Fig. 6, we measure the performance of different $\mathrm{RRC}$ pulses as a function of the truncation interval $T_{r}$, attempting to find out a suitable truncation length 


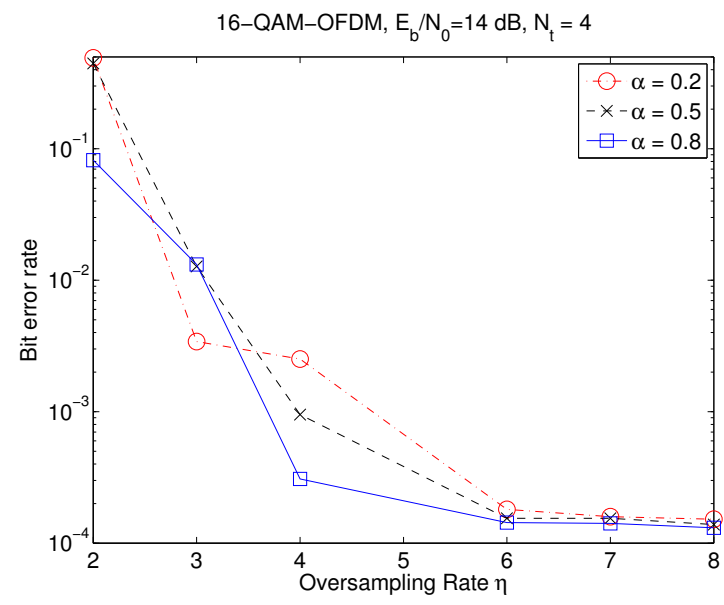

Fig. 4. The impact of oversampling rate $\eta$ on the performance of different pulses.
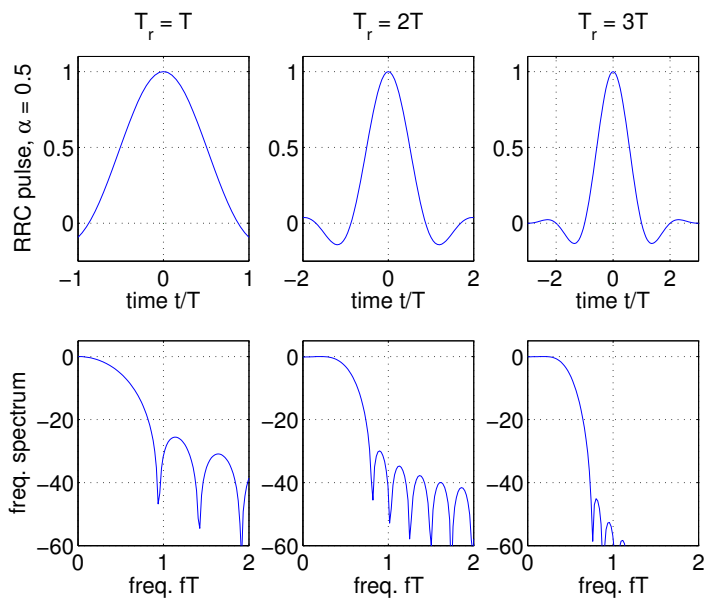

Fig. 5. The impact of truncation on signal spectrum.

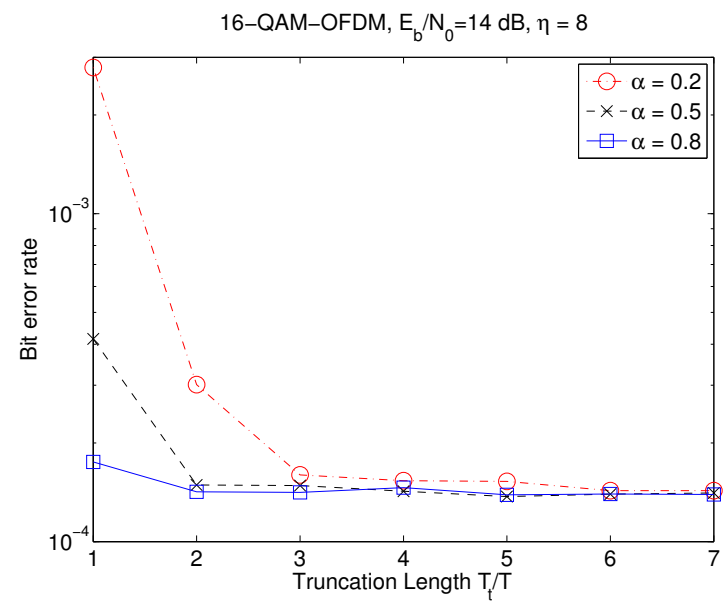

Fig. 6. The impact of truncation on the performance of different pulses.

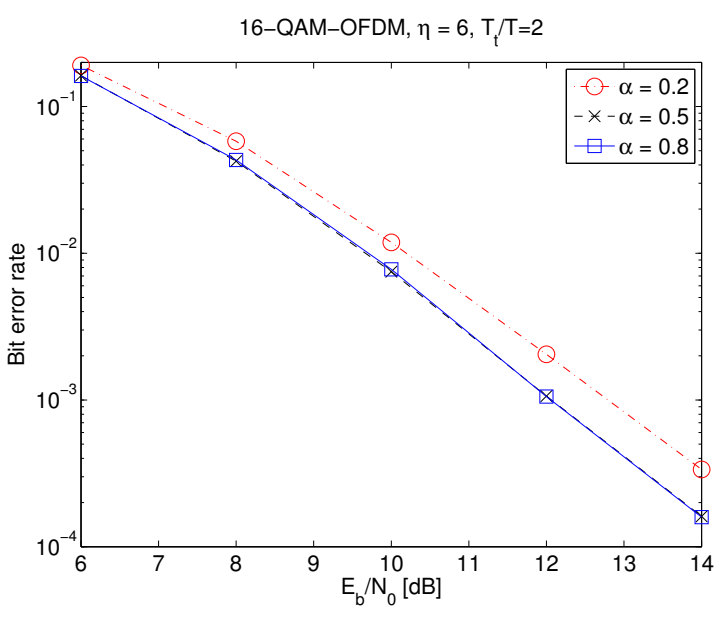

Fig. 7. Performance of different RRC pulses.

for different pulses. As expected, the RRC pulses with smaller $\alpha$ value need longer truncation interval, and vice versa. A truncation length of $T_{r}=2 T$ would suffice for the RRC pulses with rolloff factor $\alpha=0.5$ and $\alpha=0.8$. Generally speaking, $T_{r}=3 T$ would be sufficient for all RRC pulses.

In Fig. 7, we examine the performance of different RRC pulses as a function of signal to noise ratio (SNR). The truncation length is set to be $T_{r}=2 T$ for all the pulses. As indicated by the figure, the RRC pulses with $\alpha=0.5$ and $\alpha=0.8$ have almost identical performance, both perform better than the pulse with $\alpha=0.2$. The performance gap is more obvious at high SNRs. This is due to the fact that in the high SNR region, the performance is mainly influenced by ISI because noise is weak. The RRC pulses with low rolloff factor (e.g., $\alpha=0.2$ ) introduce more ISI due to larger out-of-band power caused by truncation, therefore perform worse than the ones with higher rolloff factor (e.g., $\alpha=0.5,0.8$ ). When SNR increases, ISI becomes more and more dominant, the distinction becomes clearer.

Fig. 8 shows the impact of the number of quantization bits used for each filter coefficient on the performance different pulses. It can be seen from the figure that 8 bits are needed for the RRC pulses with $\alpha=0.2$ and $\alpha=0.5$, whereas only 7 bits are needed for the RRC pulse with $\alpha=0.8$.

Figs. 9 - 11 show the signal power spectra for the WLAN system employing RRC pulses with different rolloff factors. As analyzed earlier, the pulses with higher rolloff factor value benefit from lower filter complexity and robustness against synchronization errors. However, as indicated by these figures, the signal bandwidth becomes wider as the rolloff factor increases. This complexity-bandwidth tradeoff needs to be considered in practical WLAN systems. 


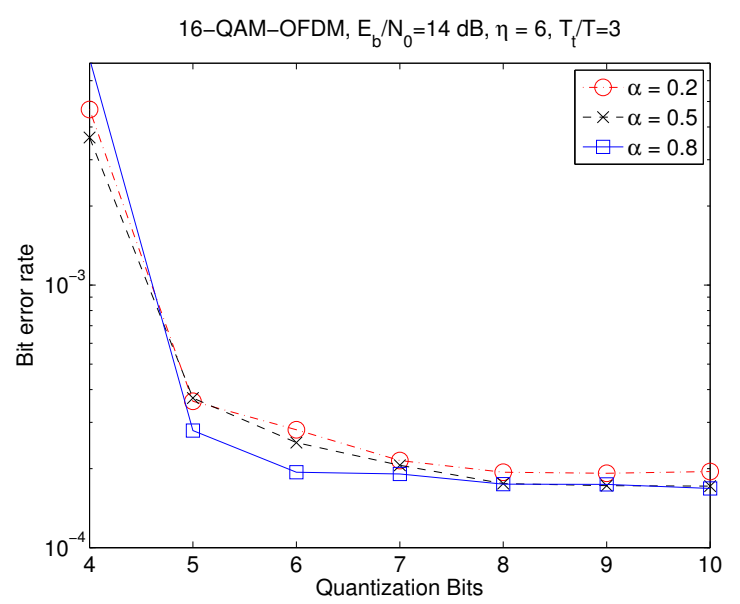

Fig. 8. The impact of the quantization on the performance of different pulses.

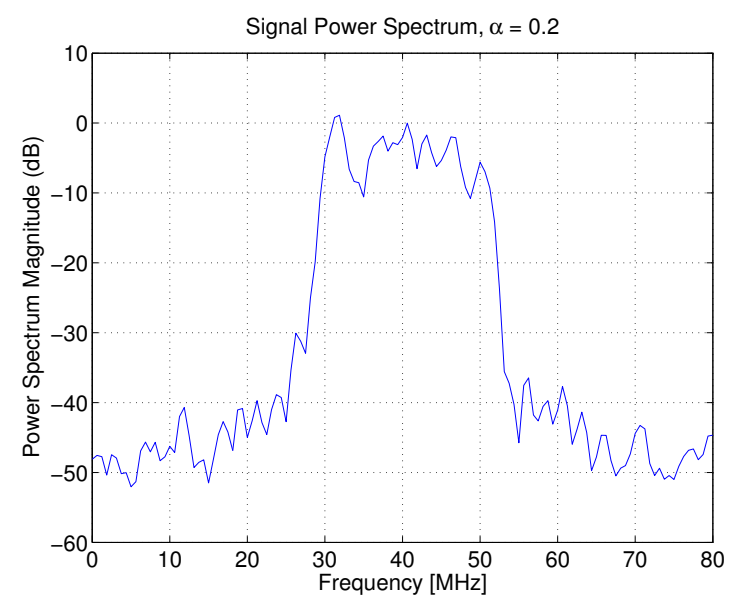

Fig. 9. Signal spectrum with RRC pulse $(\alpha=0.2)$.

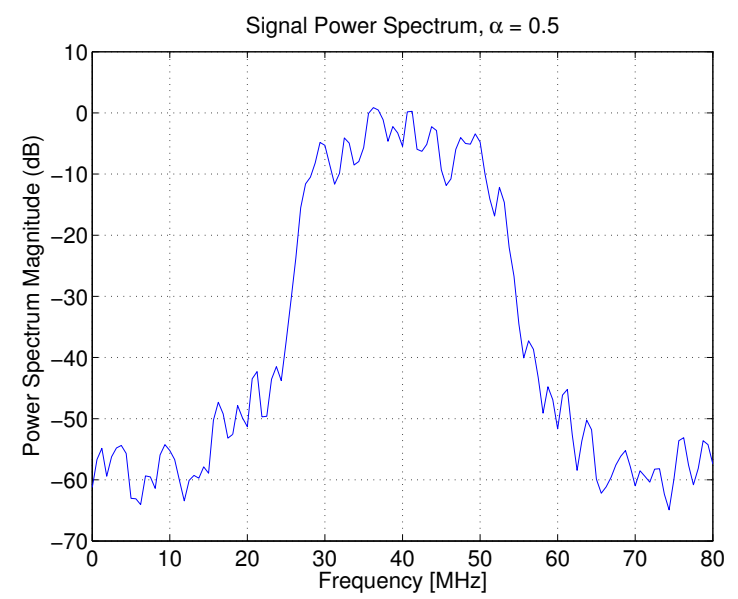

Fig. 10. Signal spectrum with RRC pulse $(\alpha=0.5)$.

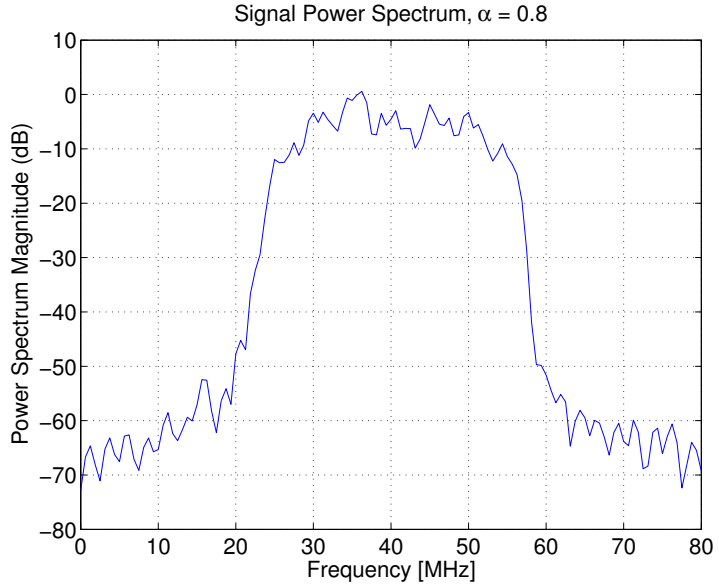

Fig. 11. Signal spectrum with RRC pulse $(\alpha=0.8)$.

\section{COnClusions}

The selection of transmit and receive filters affects not only the bandwidth efficiency, but also the performance of a WLAN system. Pulse filters with higher rolloff factor value needs shorter truncation, less quantization bits and is more resistant to synchronization errors. However, the price to pay is bigger excess bandwidth. In general, an oversampling rate of 6 , quantization bits 7 or 8 are needed for the investigated WLAN system. A truncation length of $3 \mathrm{~T} / 2 \mathrm{~T}$ on each side of the peak is needed for filter with rolloff factor of 0.2. Whereas a truncation length of $2 \mathrm{~T}$ is needed for filters with rolloff factor of 0.5 and 0.8 . With an oversample rate of 6 , it implies that 37 filter coefficients are needed in the former case; whereas 25 filter coefficients are needed in the latter case.

\section{ACKNOWLEDGEMENT}

This work was supported by the Strengthen All Island (SAI) - Mobile Wireless Future project.

\section{REFERENCES}

[1] IEEE Std 802.11a-1999, Part 11: Wireless LAN Medium Access Control (MAC) and Physical Layer (PHY) Specifications - High-speed Physical Layer in the 5 GHz Band, IEEE, 1999.

[2] R. Nee, R. Prasad. OFDM for Wireless Multimedia Communications. Artech House, 2000.

[3] 3G TS 25.104. 3rd generation partnership project: technical specification group radio access networks; $B S$ radio transmission and reception (FDD), V4.4.0, March 2002.

[4] 3GPP TR 25.814 V7.0.0. Physical layer aspects for evolved UTRA, Tech. Rep., June 2006.

[5] J. Proakis. Digital Communications, 3rd edition, McGrawHill, 1995.

[6] B. O'Hara, A. Petrick. IEEE 802.11 Handbook: A Designer's Companion. 2nd Edition, IEEE, 2005. 